\title{
Report on the 2019 Lattice Diversity and Inclusivity Survey
}

\section{Lattice Diversity and Inclusivity Committee*}

\section{Christopher Aubin}

Department of Physics and Engineering Physics, Fordham University, Bronx, NY 10458, USA

E-mail: caubin@fordham.edu

\section{Gunnar Bali}

Institut für Theoretische Physik, Universität Regensburg, 93040 Regensburg, Germany

E-mail: gunnar.balieur.de

\section{Luigi Del Debbio}

The Higgs Centre for Theoretical Physics, University of Edinburgh, Edinburgh EH9 3FD, UK E-mail: luigi.del.debbioded.ac.uk

\section{William Detmold}

Center for Theoretical Physics, Massachusetts Institute of Technology, Cambridge, MA 02139, USA

E-mail: wdetmoldemit.edu

\section{Vera Gülpers}

School of Physics and Astronomy, University of Edinburgh, Edinburgh EH9 3JZ, UK

E-mail: Vera.Guelpers@ed.ac.uk

\section{Sophie Hollitt}

Fakultät Physik, Technische Universität Dortmund, 44221 Dortmund, Germany

E-mail: sophie.hollittetu-dortmund.de

\section{Huey-Wen Lin}

Department of Physics and Astronomy, Michigan State University, East Lansig, MI 48824, USA

E-mail: hwlin@pa.msu.edu

\section{Liuming Liu}

Institute of Modern Physics, Chinese Academy of Sciences, Lanzhou 730000, China

E-mail: liuming@impcas.ac.cn

\section{Sinéad M. Ryan}

School of Mathematics, Trinity College Dublin 2, Ireland

E-mail: ryan@maths.tcd.ie

37th International Symposium on Lattice Field Theory - Lattice2019

16-22 June 2019

Wuhan, China

\footnotetext{
*Speaker.
} 


\section{Overview}

In 2018, the International Advisory Committee for the 2019 Lattice conference supported a proposal to form a Diversity and Inclusivity committee. The elected members of this committee were then charged with writing a code of conduct - which all participants agreed to as part of the conference registration; providing guidelines for conference session chairs; and lastly conducting a survey to assess diversity and inclusivity in the Lattice community and presenting the results in the conference poster session and in these proceedings.

The survey was undertaken using a Google form (and a China-based equivalent) and invitations were emailed to the attendee list from Lattice 2018 and to the latticenews mailing list. The poll was open for approximately one month and a follow-up invitation was sent with a week to go. All questions included a free-response box for answers not included in the multiple-choice options as well as a "Prefer not to answer."

There were 174 responses to the survey, many of which provided very thoughtful input. Half of the responses came from Europe, 1/3 from the US and the remaining 1/6 from Asia/Oceania/Africa.

We find that the field is not particularly diverse and is (unsurprisingly) dominated by Caucasian/white heterosexual males. The proportion of male respondents was $83 \%$, of female respondents was $11 \%$, and $3 \%$ identified as gender fluid/non-binary. The percentage of female respondents is consistent with the average rate of participation at recent lattice conferences. The rate of transgender identification was $2 \%$. The rate of respondents identifying as gay (4\%) is within the range of expectations based on similar surveys $[1,2,3,4,5]$.

There are issues with inclusivity in the community; $25-30 \%$ of respondents indicated not feeling comfortable infrequently or more often at talks, social events and even conference accommodations. A significant number of respondents (about 35\%) has observed or had negative experiences. The nature of these experiences was not determined in the survey.

Histograms of responses to survey questions based on different demographic data collected are shown below in Sec. 3. For the free text questions, results are summarised here but explicit responses are not included in this write-up.

Respondents felt the gender balance of plenary speakers has improved. This impression is not supported by the statistics of the last ten conferences. Respondents felt that the percentage of women in the field was higher than it actually is. Respondents are generally supportive of efforts to improve diversity (e.g., a diversity committee, 2018 lunch talks). Respondents felt the tone of discussions has become less aggressive in comparison to the 90's. Future surveys should probe more detailed reasons for the discomfort or negative experiences of the respondents.

In these proceedings we discuss recommendations based on the results of the survey in Sec. 2. In Sec. 3 we show the results from the questions regarding comfort levels and negative experiences from the survey, and we briefly conclude in Sec. 4. We include the responses to the demographics questions in Appendix 1 and an update of the Women in Lattice statistics in Appendix 2.

\section{Recommendations}

After reviewing the results from the survey, the committee has the following recommendations for the lattice community. 
1. Formalize the diversity committee and maintain the code of conduct. Survey diversity periodically, updating the questionnaire based on the lessons learned from this survey and keep statistical records.

2. Continue the "Women in Lattice" lunches, opening this event to any interested participants.

3. Regularly hold a plenary on the topic of diversity and inclusivity.

4. Further increase the level of diversity in plenary talks and session chairs (not just monitoring the gender balance).

5. Provide financial support for minorities as well as for students.

6. Foster mentoring of junior colleagues. Identify senior physicists to meet with a group of younger scientists for career discussions.

7. Attempt to promote international mixing between participants at different career stages.

8. Establish a clear mechanism for reporting and responding to incidents of exclusion and harassment.

9. Develop an informal practical guide for future local organizing committees, related to diversity and inclusivity, to help ensure a positive atmosphere for all participants. This should be updated and passed on from year to year, along with the lattice conference manual.

\section{Summary of the Survey Results}

The following histograms correlate the questions "Did you feel comfortable at the most recent lattice conference you attended?" and "Have you had direct or indirect (personally or observed) negative experiences at a lattice conference?" with various demographics questions. We have averaged each person's responses over the different parts of the conference that were asked about in the survey (such as "during scientific talks" or "during social events") for simplicity. Additionally, we have normalized each response by the total number of respondents in that category (such as "male" or "female") for easier comparison. In all of the histograms below, we have made the following abbreviations for the labels
A: Always
F: Frequently
S: Sometimes
I: Infrequently
N: Never
N/A: Not applicable

\subsection{Age}

Given the few responses in the age category " $>70$ " we have combined those with the $61-70$ age group. We show in Fig. 1 the answers to these questions by age. 


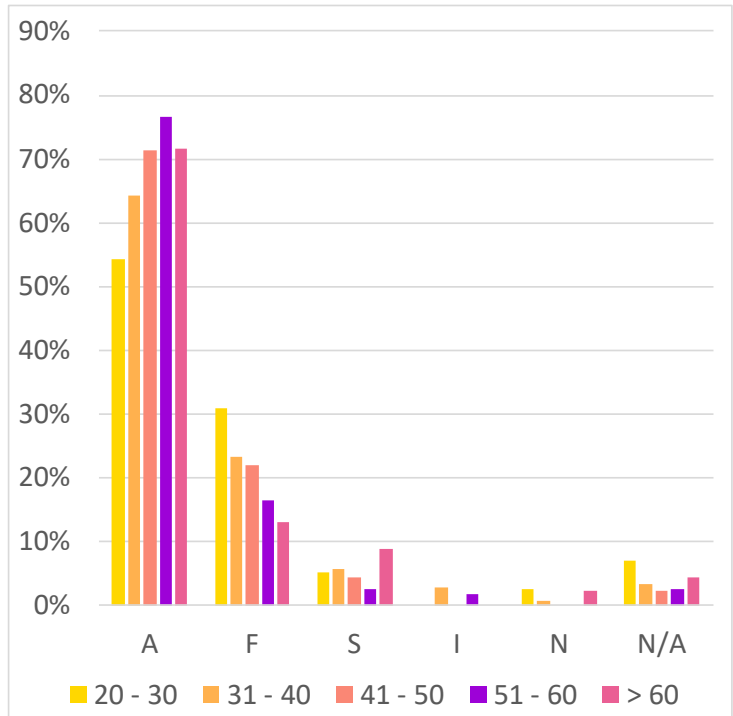

(a)

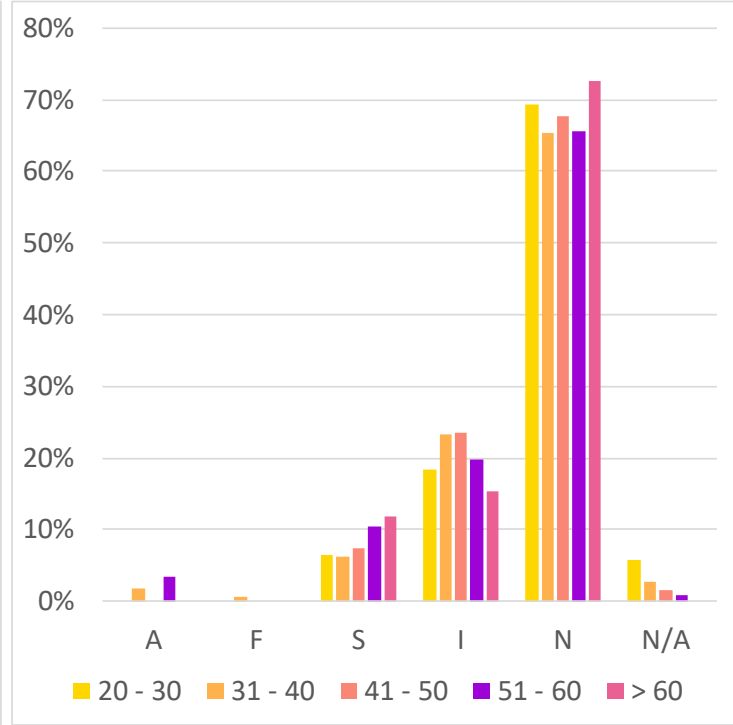

(b)

Figure 1: Answers to the following questions by age: (a) "Did you feel comfortable at the most recent lattice conference you attended?" (b) "Have you had direct or indirect (personally or observed) negative experiences at a lattice conference?"

\subsection{Rank/Position}

Figure 2 presents the responses to the academic climate questions with respect to academic rank. In these categories the "Other" category includes the responses: Industry, Editor, Adjunct, Untenured faculty, Former Graduate Student, and those who selected "Prefer not to answer." Here it seems as though lack of comfort/more negative experiences are seen by those in the "Retired" group, and to a lesser extent the "Graduate Students."

\subsection{Gender Identity}

For the plots in Fig. 3, we make three categories in order to not just compare responses between those who identify as male or female but also those who do not identify as male. This latter category includes all responses that were not male: female, gender fluid/non-binary, write-in responses and "prefer not to answer." It is clear that negative experiences are more common for those who do not identify as male.

\subsection{LGBTQIA Responses}

In Fig. 4, we look at responses from those who do not identify as heterosexual as well as those with any LGBTQIA (Lesbian-Gay-Bisexual-Trans-Queer-Intersex-Asexual) identity (broken up into those who are mostly "out" to the lattice community and those who are not). Those who are out are less likely to have felt uncomfortable or to have had negative experiences.

Additionally in Fig. 5, we examine the responses from those who think that their identity (be it gender identity or sexual orientation) is known by others in the lattice community. Those whose identities are known appear to feel more comfortable at the lattice conferences and are slightly less 


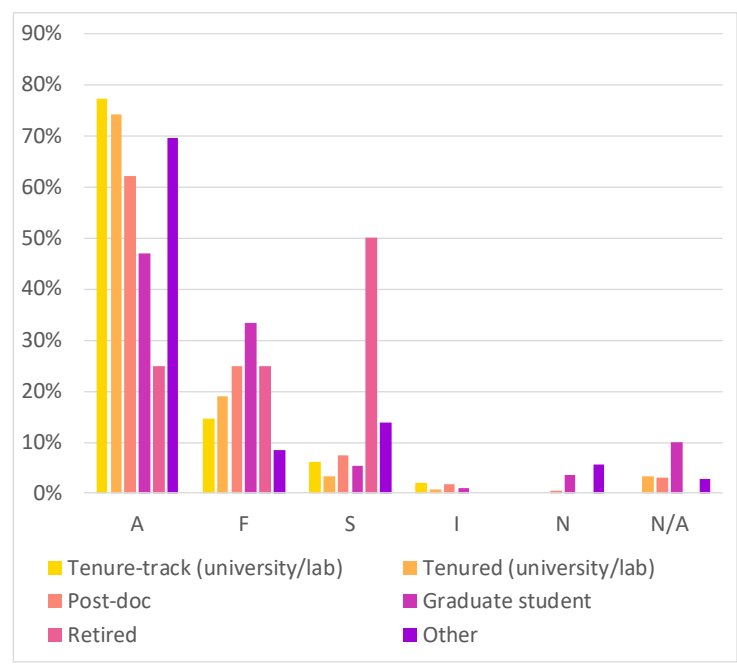

(a)

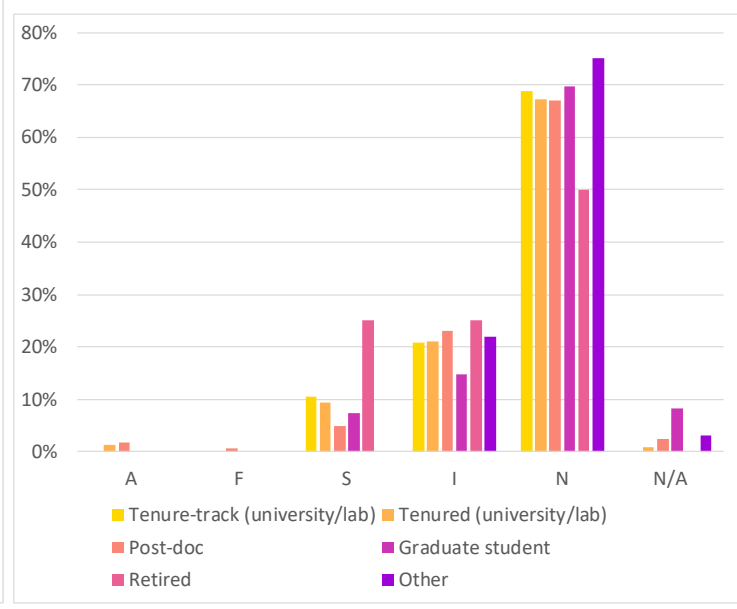

(b)

Figure 2: Answers to the following questions by rank: (a) "Did you feel comfortable at the most recent lattice conference you attended?" (b) "Have you had direct or indirect (personally or observed) negative experiences at a lattice conference?"

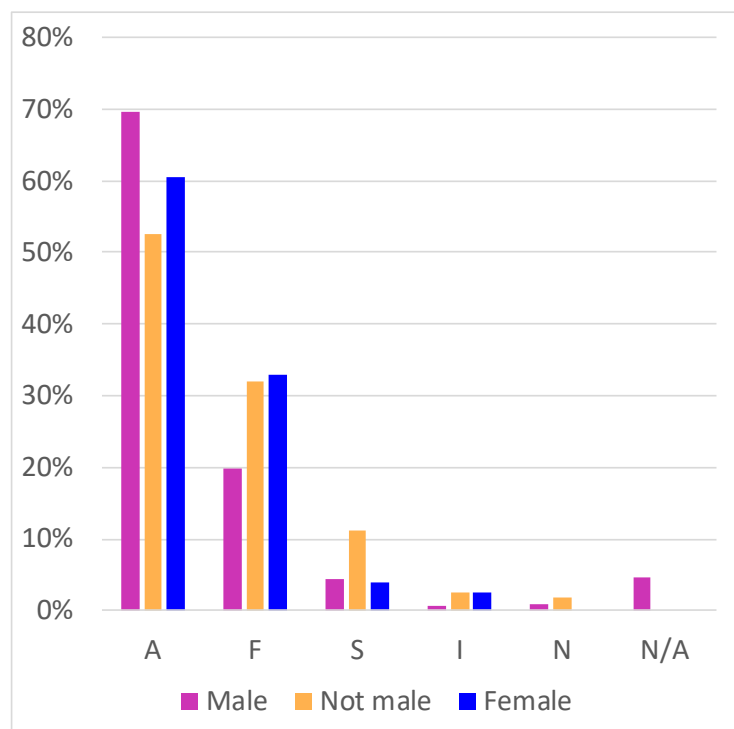

(a)

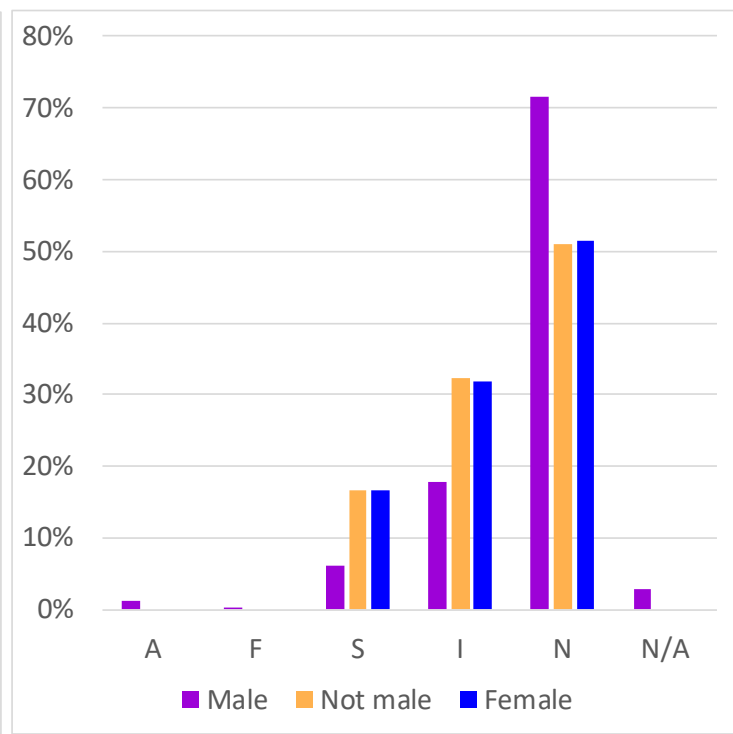

(b)

Figure 3: Answers to the following questions by gender identity: (a) "Did you feel comfortable at the most recent lattice conference you attended?" (b) "Have you had direct or indirect (personally or observed) negative experiences at a lattice conference?" 


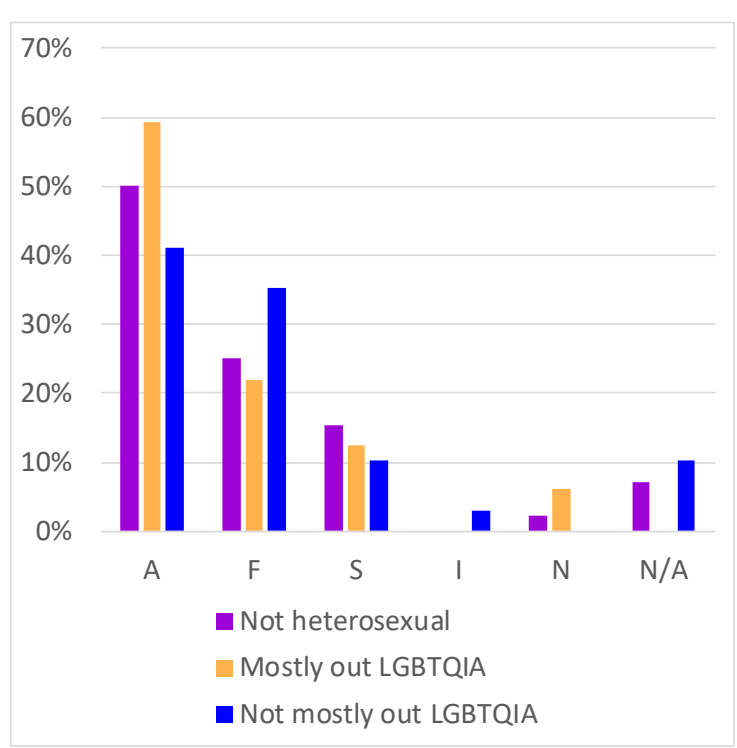

(a)

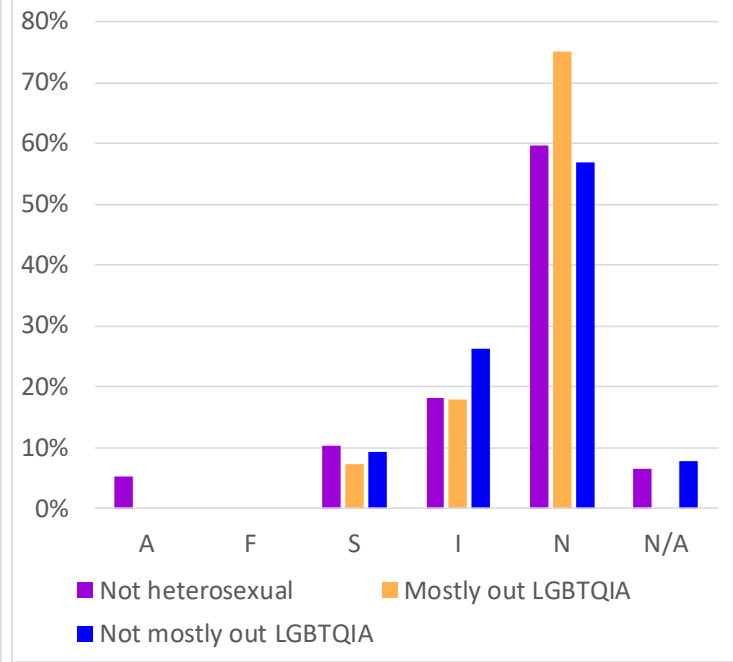

(b)

Figure 4: Answers to the following questions by LGBTQIA identity: (a) "Did you feel comfortable at the most recent lattice conference you attended?" (b) "Have you had direct or indirect (personally or observed) negative experiences at a lattice conference?"

likely to have had negative experiences. This makes it clear that we should work on having a more positive and inclusive atmosphere.

\subsection{Comfort Level and negative experiences during conferences}

In Table 1(a), we show the raw numbers from responses to the question "Did you feel comfortable at the most recent lattice conference you attended?" For this table, 1 is "never comfortable" and 5 is "always comfortable" at various times during the conference. (In both tables, "Accomm." stands for Accommodations.)

Talks Events Breaks Accom.

\begin{tabular}{ccccc}
\hline \hline 1 & 2 & 2 & 2 & 0 \\
\hline 2 & 2 & 2 & 2 & 0 \\
\hline 3 & 6 & 13 & 13 & 6 \\
\hline 4 & 33 & 40 & 43 & 34 \\
\hline 5 & 125 & 111 & 108 & 114 \\
\hline $\mathrm{n} / \mathrm{a}$ & 4 & 4 & 4 & 15 \\
\hline
\end{tabular}

(a)
Talks Events Breaks Accom.

\begin{tabular}{ccccc}
\hline \hline 1 & 95 & 113 & 118 & 136 \\
\hline 2 & 48 & 32 & 34 & 23 \\
\hline 3 & 21 & 19 & 12 & 0 \\
\hline 4 & 0 & 0 & 0 & 0 \\
\hline 5 & 2 & 0 & 2 & 2 \\
\hline $\mathrm{n} / \mathrm{a}$ & 3 & 3 & 3 & 8 \\
\hline
\end{tabular}

(b)

Table 1: In (a), 1 is "never comfortable" and 5 is "always comfortable" at various times during the conference. In (b), 1 is "never had a negative experience." 5 is "always had a negative experience."

The majority of respondents always felt comfortable. However it is striking that about $25 \%$ of respondents felt uncomfortable even some of the time during talks, social events and even at 


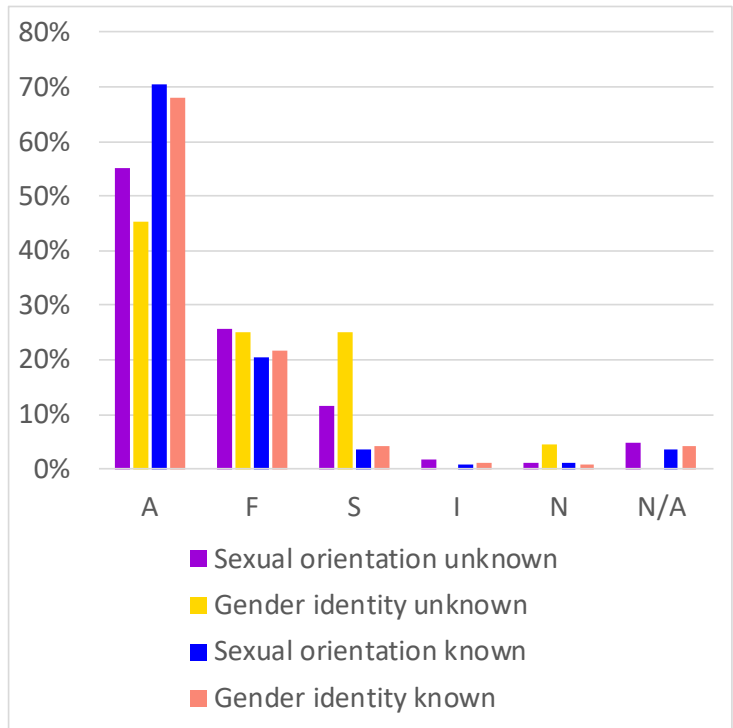

(a)

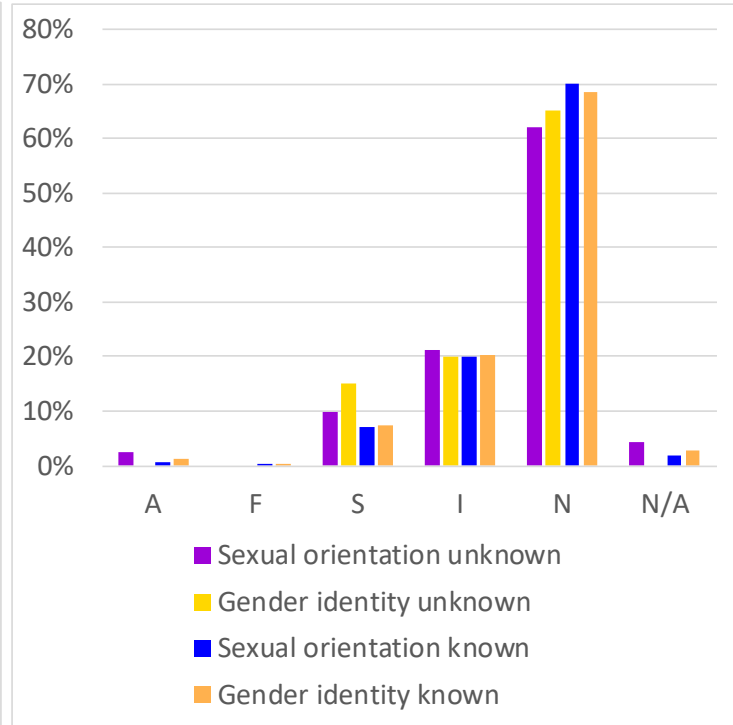

(b)

Figure 5: Answers to the following questions by perceived knowledge of LGBTQIA or gender identity: (a) "Did you feel comfortable at the most recent lattice conference you attended?" (b) "Have you had direct or indirect (personally or observed) negative experiences at a lattice conference?"

conference accommodations. The highest levels of discomfort were at social events and informal gatherings/breaks.

In Table 1(b) we show the raw numbers from responses for the question "Have you had direct or indirect (personally or observed) negative experiences at a lattice conference?" Here, 1 is "never had a negative experience." 5 is "always had a negative experience." In each case the majority did not report negative experiences, however about $35 \%$ of respondents have been subject to, or witnessed, negative experiences.

\section{Conclusion}

As discussed above, in general the community is amenable to programs such as this committee and other ways to improve diversity in the field. Steps should be taken in order to (as much as possible) improve inclusivity for all participants at all conferences.

The IAC has approved our proposal to form a standing Diversity and Inclusivity Committee. This Committee will update the Code of Conduct, promote diversity and inclusivity in the community and periodically survey the progress made. We ask that any suggestions for improvement are forwarded to the members of this Committee.

\section{Appendix 1: Demographics Questions}

In this appendix we include graphs showing the direct answers to the demographics questions in the survey. 


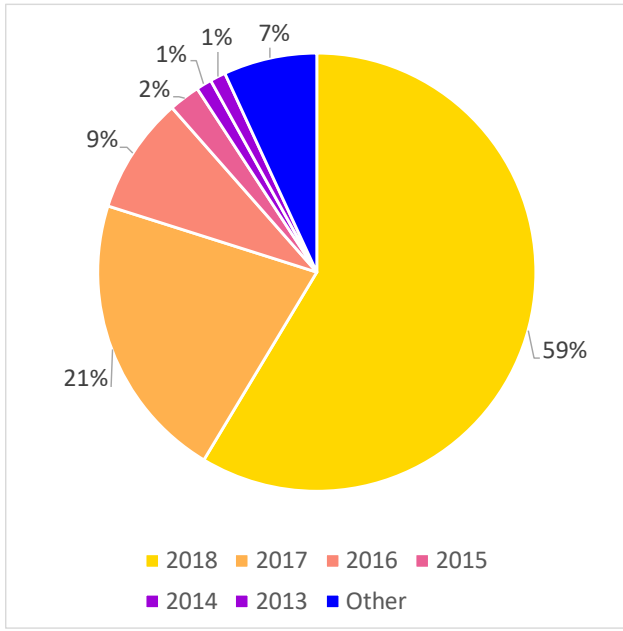

(a)

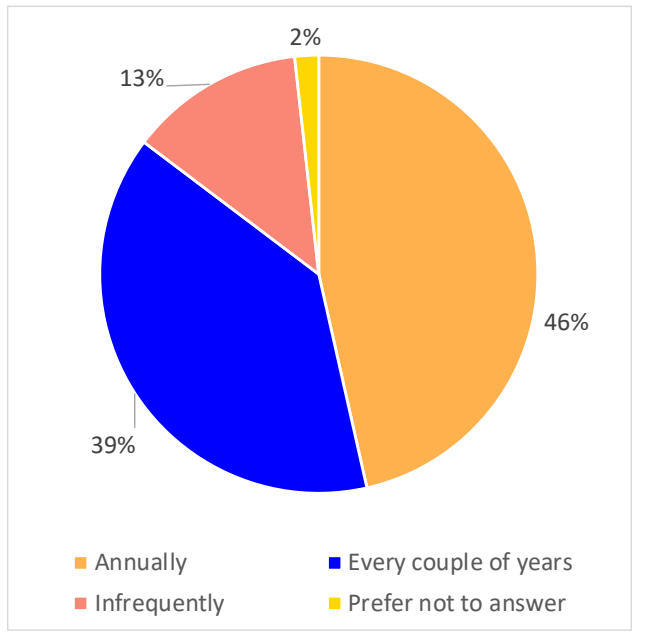

(b)

Figure 6: (a) What year was the last lattice conference you attended? (b) How often do you attend the lattice conference?

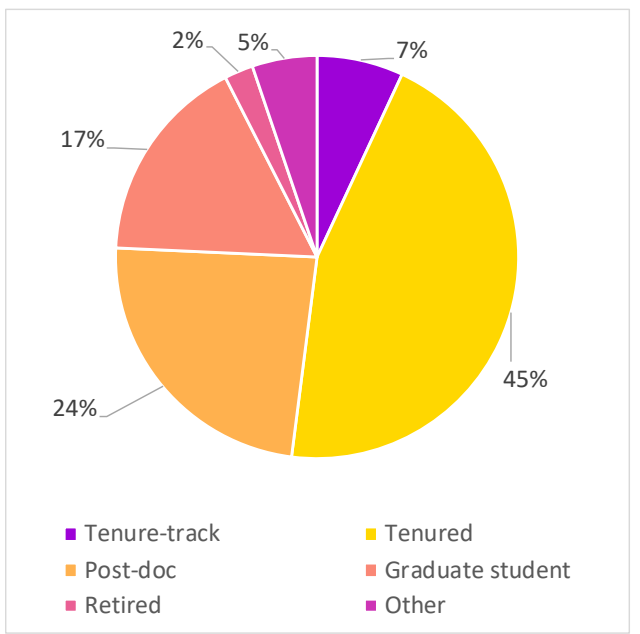

(a)

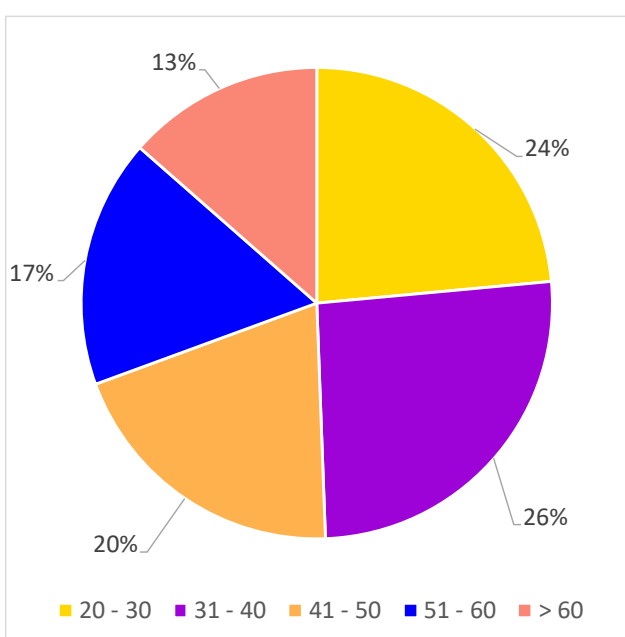

(b)

Figure 7: (a) What is your current rank? (b) What is your age?

\section{Appendix 2: Women in Lattice Statistics (Updated)}

The fraction of female participants has increased relative to the previous decade, as can be seen in Fig. 11. This is likely correlated with a global trend of increasing PhD-degrees obtained by female students. We can compare this with the question on the LDIC survey which asked respondents to estimate the percentage of women at the last lattice conference they attended. About one-third of respondents guessed in the 5-10\% range, which was true in most of the conferences in the last ten years. About one-third assumed there were 10-15\% female participation which is only true in three conferences $(2013,2016$, and 2017). $28 \%$ of survey participants chose a response 


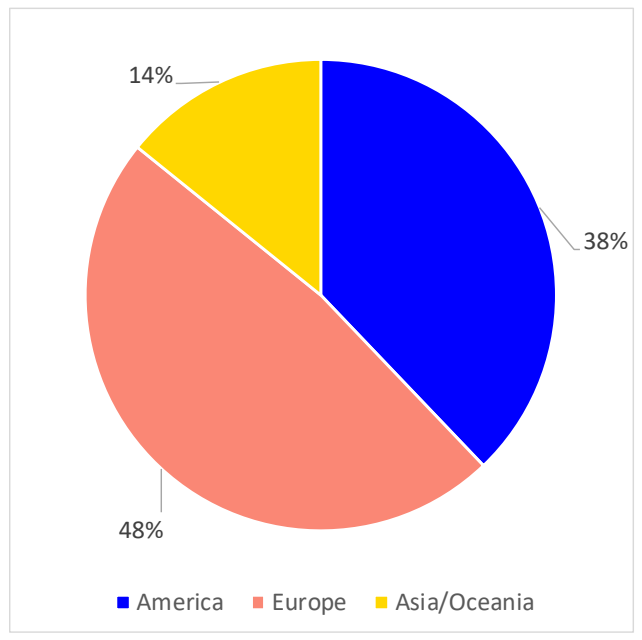

(a)

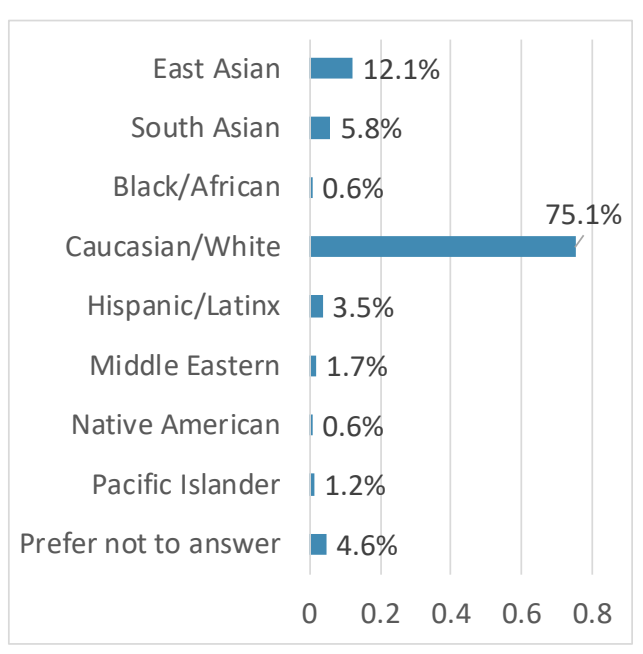

(b)

Figure 8: (a) What geographic region do you work in? (b) What is your ethnicity?

that was $>15 \%$, showing that a significant number of participants easily overestimate the number of female participants at the conference.

It would be interesting to cross-check the observed trend with the recent APS data. Conferences in the EU continue to have larger fractions of female participation. Are there lessons that US/Asia can learn to improve the female participant rates or is this due to culture/grants limitations?

In Fig. 12 we show the percentage of female plenary speakers at the lattice conference (purple) and the APS April Meeting (orange) as a function of year. Most of lattice conference has around $10 \%$ of female speakers. In comparison with APS April meeting, which covers similar fields in nuclear and particle physics, there is room for much improvement in Lattice QCD.

Finally we show the number of male and female plenary speakers as a function of the number of times those speakers were invited in Fig. 13. There is a roughly $10 \%$ fraction of women in our field (in contrast with APS: 30\%). Can the small number of plenary talks given by women be due to lack of women in our field? There is a considerably stronger tendency to re-invite male speakers for plenary talks, rather than to re-invite past female speakers.

More updated statistics can be found online in this google directory.

\section{References}

[1] T. J. Atherton, R. S. Barthelemy, W. Deconinck, M. L. Falk, S. Garmon, E. Long, M. Plisch, E. H. Simmons, and K. Reeves, LGBT Climate in Physics: Building an Inclusive Community, American Physical Society (2016).

[2] H.-W. Lin, Some Statistics on Women in Lattice QCD, PoS LATTICE2016 (2017) 366, [arXiv:1612.0936].

[3] B. Beckford, Strategies to improve diversity and inclusion in physics, Talk given at the XXXIX International Conference on High Energy Physics (ICHEP 2018) (2018). 


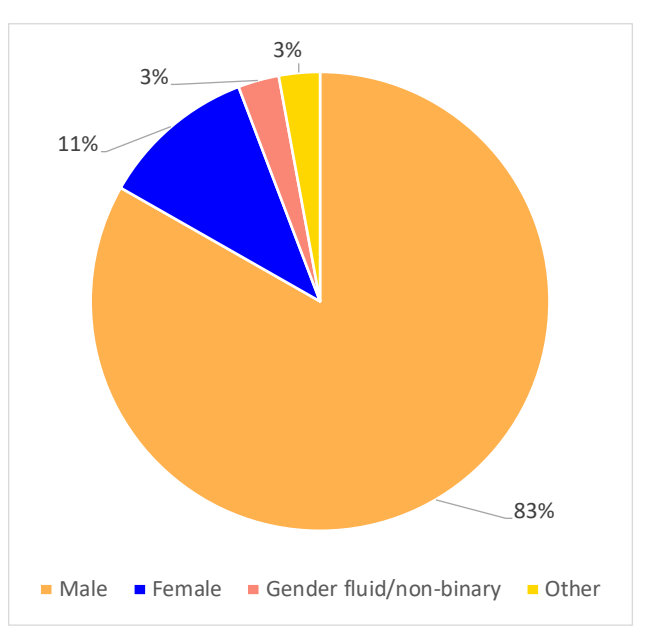

(a)

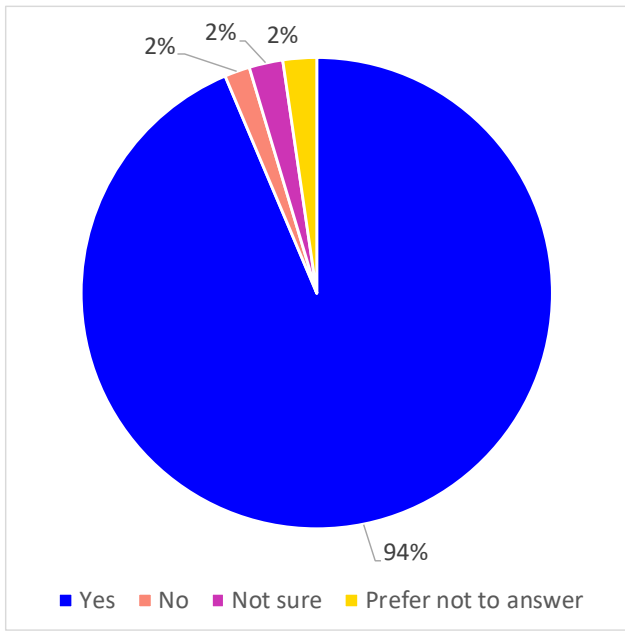

(b)

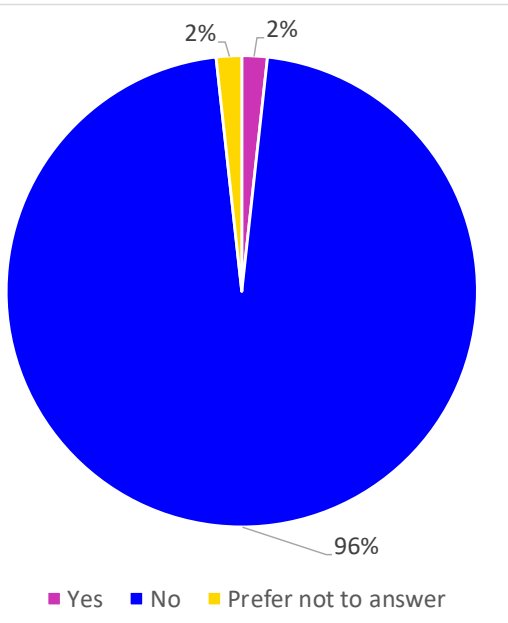

(c)

Figure 9: (a) What best describes your gender identity? (b) Do you think most people are aware of your gender identity? (c) Do you identify as transgender?

[4] S. Yacoob, Diversity \& Inclusion, Talk given at the XXXIX International Conference on High Energy Physics (ICHEP 2018) (2018).

[5] L. M. Aycock, Z. Hazari, E. Brewe, K. B. H. Clancy, T. Hodapp, and R. M. Goertzen, Sexual harassment reported by undergraduate female physicists, Phys. Rev. Phys. Educ. Res. 15 (Apr, 2019) 010121. 


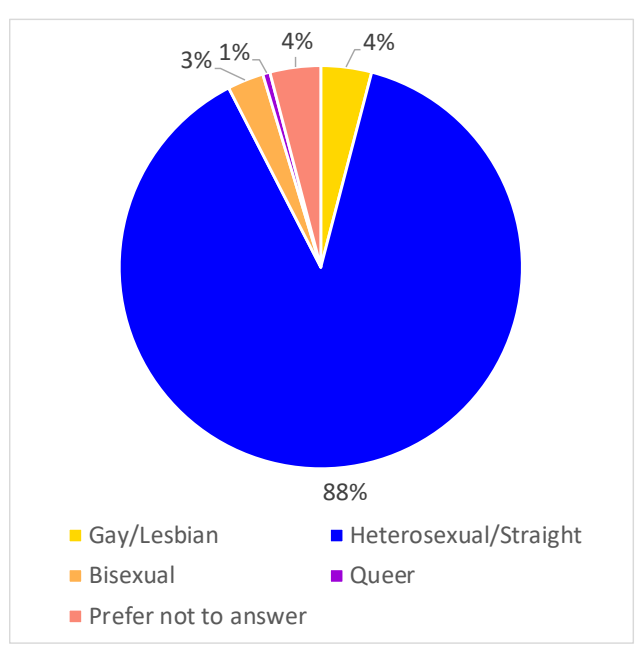

(a)

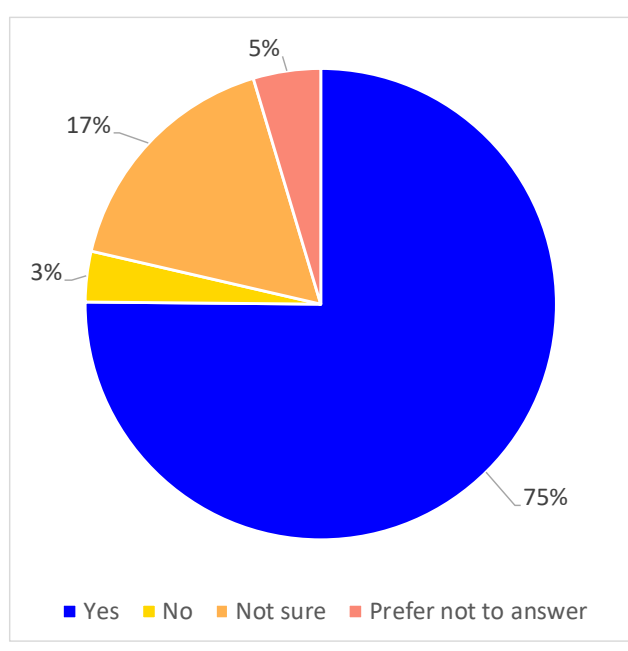

(b)

Figure 10: (a) What is your sexual orientation? (b) Do you think most people are aware of your sexual orientation?

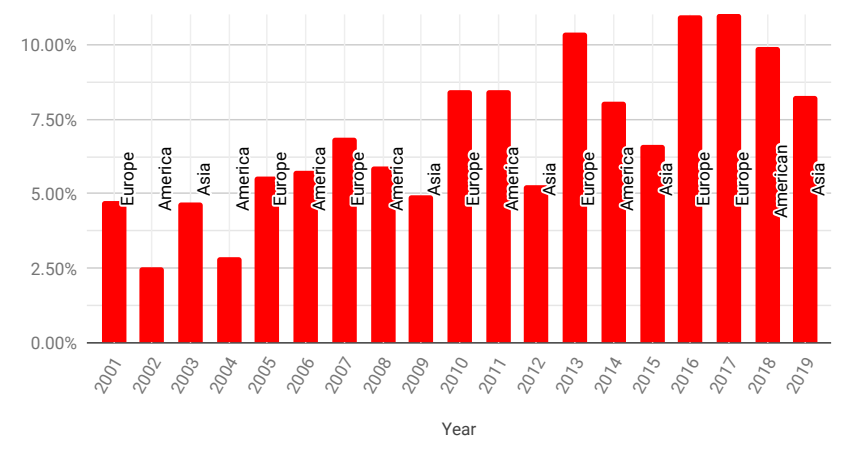

(a)

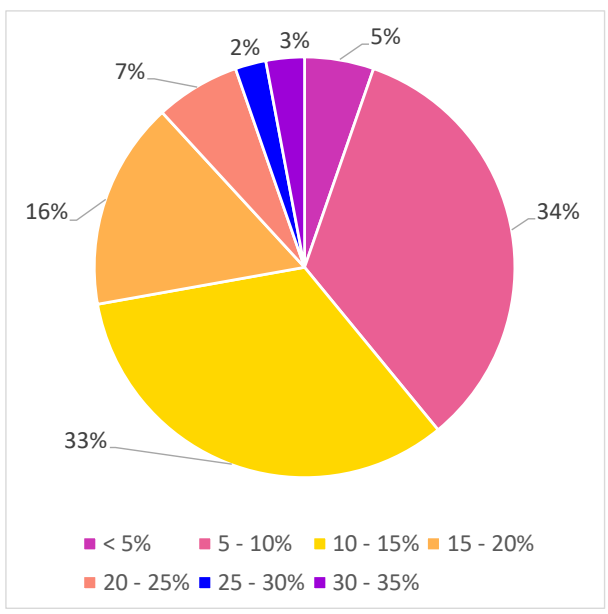

(b)

Figure 11: (a) Women fraction of lattice conference participation, compared with (b) the fraction of women perceived to be in attendance by respondents to the LDIC survey. 


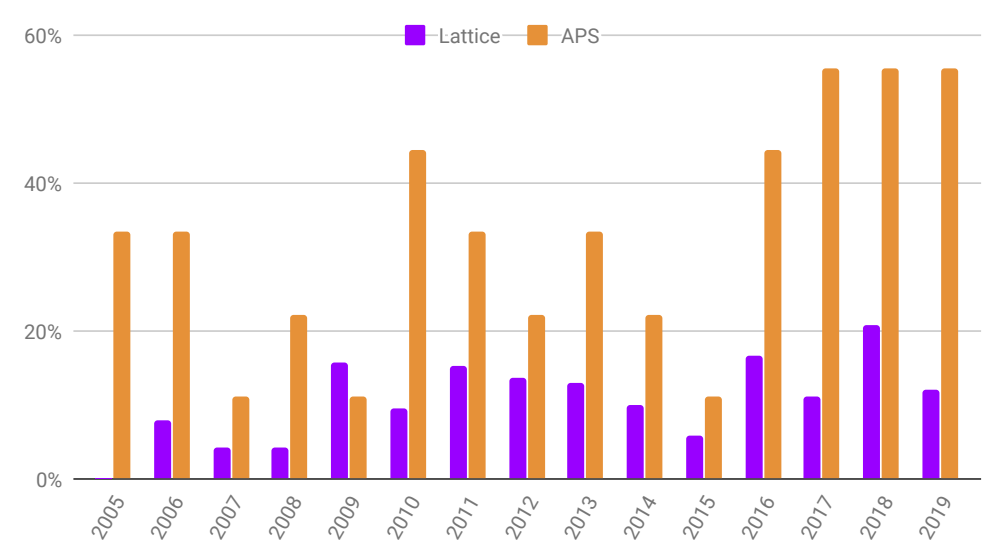

Figure 12: Comparison of the fraction of women plenary speakers between lattice conferences and the April APS meetings.

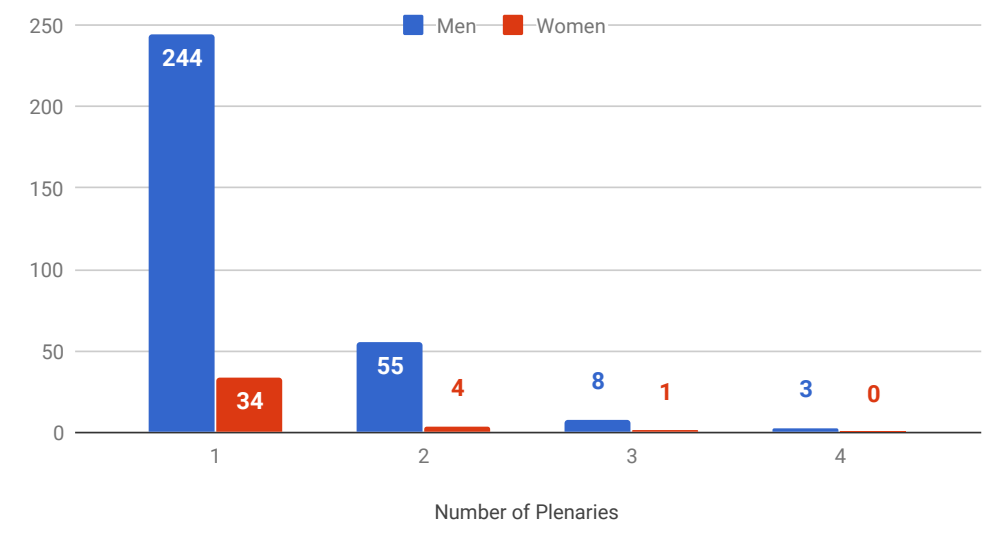

Figure 13: Lattice plenary reinvitations by gender. 